\title{
The painless combination of anatomically contoured titanium plates and porcine dermal collagen patch for chest wall reconstruction
}

\author{
Kingsfield Ong ${ }^{1}$, Chin Siang Ong ${ }^{2}$, Yang Chong Chua ${ }^{3}$, Ali Akbar Fazuludeen ${ }^{1}$, Aneez Dokeu Basheer Ahmed ${ }^{1}$ \\ ${ }^{1}$ Division of Thoracic Surgery, Department of General Surgery, Tan Tock Seng Hospital, Singapore; ${ }^{2}$ Johns Hopkins School of Medicine, Baltimore, \\ Maryland, USA; ${ }^{3}$ Division of Surgical Oncology, National Cancer Centre, Singapore \\ Contributions: (I) Conception and design: K Ong, YC Chua, ADB Ahmed; (II) Administrative support: AA Fazuludeen, ADB Ahmed; (III) Provision of \\ study materials or patients: K Ong, AA Fazuludeen, ADB Ahmed; (IV) Collection and assembly of data: K Ong; (V) Data analysis and interpretation: \\ All authors; (VI) Manuscript writing: All authors; (VII) Final approval of manuscript: All authors. \\ Correspondence to: Aneez Dokeu Basheer Ahmed, FRCS (CTh). Division of Thoracic Surgery, Department of General Surgery, Annex Building 1, \\ Level 4, 11 Jalan Tan Tock Seng, Singapore. Email: dokeu_basheer@ttsh.com.sg.
}

\begin{abstract}
Background: A plethora of new biomaterials and dedicated rib fixator implant systems have been introduced into the field of chest wall reconstruction. The aim of our study is to evaluate the surgical outcomes of a novel combination of the anatomically contoured titanium rib implant and porcine dermal collagen patch for chest wall reconstruction.

Methods: We performed a retrospective review of eight consecutive patients who underwent chest wall resection and reconstruction between January 2014 to August 2015 in a single institution. MatrixRib Fixation System and Permacol Surgical Implant were utilized to achieve chest wall reconstruction.

Results: The indication for reconstruction was malignant infiltration in $50 \%$ of patients. Three other subjects $(37.5 \%)$ had chest wall resections to achieve adequate and safe surgical exposure. One patient had a right lung apical mycetoma with chest wall invasion. All patients underwent lung resections with the removal of 2 to 6 (median 3) ribs. Reconstruction was performed using the MatrixRib system, with a median of 2.5 (range, 2-4) ribs fixed in each patient. There was no post-operative mortality. One patient had a superficial wound infection which resolved with one week of oral antibiotics. Upon discharge, the pain scores were near zero with minimal analgesic requirements. None of the patients required repeat surgery or removal of their implants.

Conclusions: Our early experience indicates that the combination of the MatrixRib system and Permacol patch for chest wall reconstruction is safe and feasible with promising results in terms of anatomical restoration of the chest wall mechanics, infection and pain.
\end{abstract}

Keywords: Chest wall reconstruction; titanium plates; collagen patch; painless

Submitted Dec 29, 2017. Accepted for publication Apr 04, 2018.

doi: $10.21037 /$ jtd.2018.04.116

View this article at: http://dx.doi.org/10.21037/jtd.2018.04.116

\section{Introduction}

It has been established that surgical repair of large chest wall defects facilitates the restoration of physiological chest wall mechanics for normal respiration $(1,2)$. The search for the ideal reconstruction amidst a plethora of different approaches and materials is ongoing, with much debate on the preferred technique and material of choice $(3,4)$.

Over the past decade, we have observed significant success with titanium devices for rigid chest wall fixation (5-7). Soft tissue reconstruction commonly involved the utilization of synthetic materials such as polytetrafluoroethylene (PTFE) and polypropylene 
or muscle flaps (6). In fact, biomaterials for soft tissue reconstruction are gaining in popularity, boasting high strength and resistance to infection as compared to the standard PTFE or polypropylene meshes (8-10). Encouraging results have been reported in contaminated surgical environments $(11,12)$. In addition, combinations of different synthetic and bio-prosthetic materials for chest wall reconstruction have shown promising results $(1,5,13)$.

In light of these technological advances in chest wall reconstruction, we have employed the combination of titanium plates and screws (MatrixRIB ${ }^{\text {TM }}$ Fixation System, DePuy Synthes CMF, West Chester, PA, USA) and a matrix of porcine dermal collagen (Permacol ${ }^{\mathrm{TM}}$ Surgical Implant, Medtronic, Minneapolis, MN, USA) for the repair of chest wall defects at our institution. We present our initial experience with this technique of chest wall reconstruction, discuss some technical aspects of the surgical reconstruction and evaluate the postoperative outcomes in this cohort of patients.

\section{Methods}

\section{Data collection}

This is a retrospective single-institution study of 8 consecutive patients who underwent chest wall reconstruction with the novel combination of MatrixRib and Permacol from January 2014 to August 2015. Chart review was performed to obtain clinical data such as basic demographics, surgical indication, operative time, intensive care unit (ICU) stay, chest tube duration, hospital stay duration, underlying pathology, post-operative complications (within 30 days of surgery), pain scores and analgesic requirements at discharge. Specific surgical data collected included type of surgery, extent of resection, number and location of ribs resected and the number of ribs reconstructed. All patients were reviewed at the 6-month and 1-year follow-up clinic. This study has been approved by the Domain Specific Review Board National Health Care Group (Singapore), with waiver of patient consent.

\section{Surgical preparation and technique}

All patients underwent computed tomography (CT) of the chest. Patients with malignant disease had a positron emission tomography/CT scan to assess for regional and local metastasis. Comprehensive pre-operative assessment, investigations and consent were obtained in all patients.
The thoracic and plastic surgery teams collectively performed the resection and reconstruction together. All patients were put under general anesthesia with double lumen endotracheal intubation and single lung ventilation. Pain management was optimized further with thoracic paravertebral nerve block catheter intraoperatively. Surgical access was obtained via muscle sparing posterolateral thoracotomy.

Various techniques were utilized to retain the anatomical structure of the chest wall and optimize the stability of the titanium plates. From the pre-operative investigations and imaging, we identified the ribs that were to be resected. Intra-operatively, a mold was taken before the resection of the respective ribs. The MatrixRib system of titanium plates and screws were sized appropriately and fixed with at least three screws on either side of the mold, then unscrewed and kept on the operating table. Thereafter, the identified ribs were resected and the titanium plates were placed over the respective ribs accurately to maintain the anatomical structure of the chest wall. Stability was achieved by having a minimum of $3 \mathrm{~cm}$ overlap between the remaining edge of the resected rib and titanium plates, with at least three screws on either side. Soft tissue reconstruction was achieved in all patients using a single layer of Permacol patch. The Permacol patch was trimmed to fit and secured under tension with interrupted non-absorbable sutures (Prolene 2-0) to the pericostal edges of the chest wall defect, on top of the titanium plates. To drain the thoracic cavity, a 28 or $32 \mathrm{~F}$ chest tube was inserted and connected to a digital closed-system chest drainage and suction unit (Thopaz Digital Chest Drainage System, Medela, Baar, Switzerland). There were no additional drains placed over the prostheses. Prophylactic antibiotics were administered for 48 hours post-operatively. Patients who had pre-existing lung infections had antibiotics continued as required.

\section{Results}

There was a total of 8 consecutive patients enrolled in this study ( 6 males, 2 females), with a mean age of 61.5 years (range, $43-75$ years) (Table 1).

Indications for surgery in relation to their pathology were malignant disease $(n=4,50 \%)$, persistent hemoptysis with superimposed infection ( $\mathrm{n}=3$, bronchiectasis, mycetoma and tuberculosis) and one ventilator dependent patient had a right bronchus intermedius stricture and malacia with persistent collapse of right middle and lower lobe (Table 2).

Indications for chest wall resection and reconstruction 


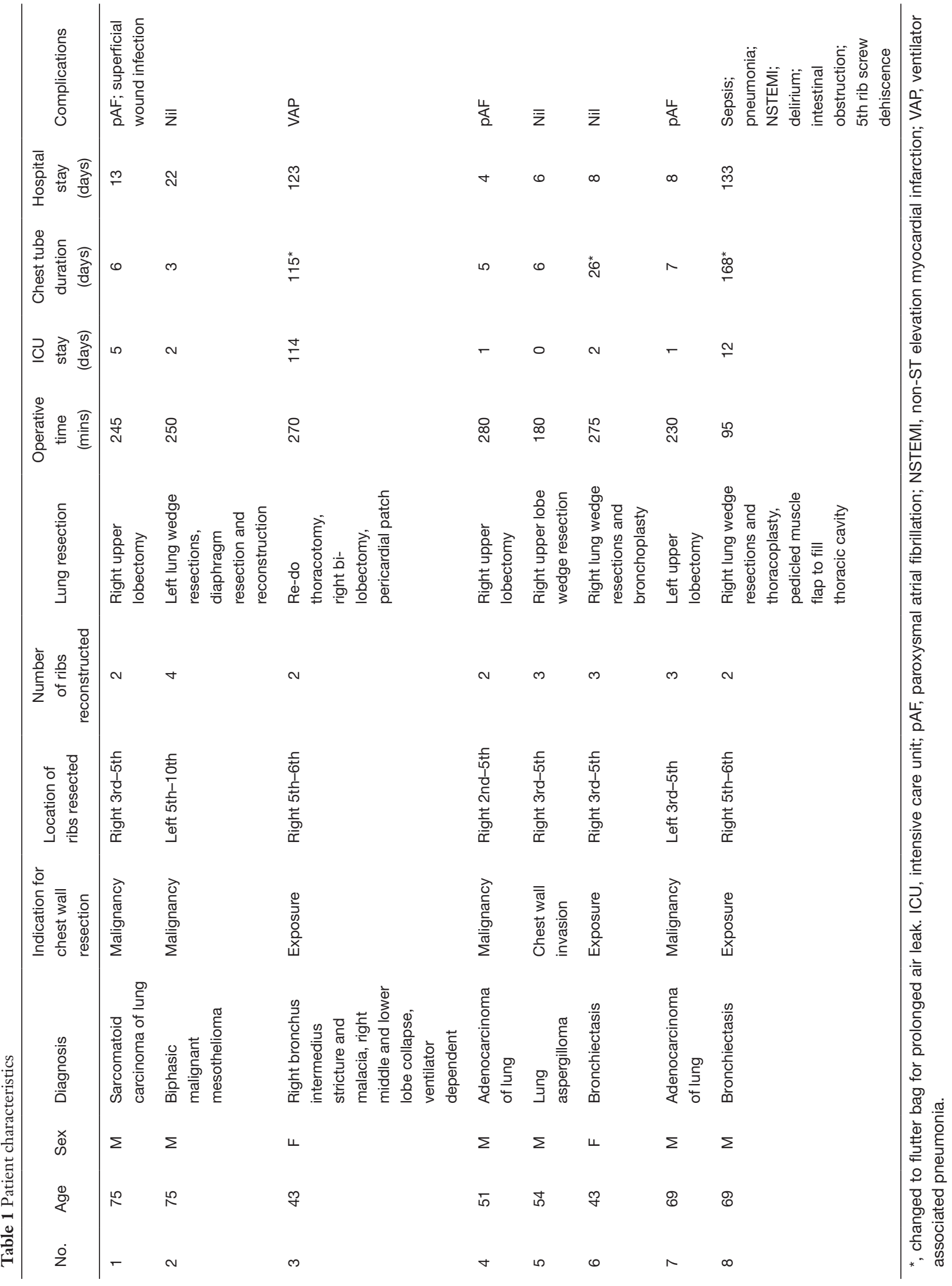


Table 2 Clinical summary

\begin{tabular}{lc}
\hline Characteristics & $\mathrm{N}=8$ \\
\hline Gender (M:F) & $6: 2$ \\
Age $^{\star}$ & $61.5[43-75]$ \\
Side (R:L) & $6: 2$ \\
Indications for chest wall resection & \\
Malignancy & 4 \\
Exposure & 3 \\
Chest wall invasion & 1 \\
Post-op wound infection & \\
Superficial & 1 \\
Deep & 0 \\
Operative time (mins) & \\
Chest tube duration (days) & $247.5[95-280]$ \\
Hospital stay (days) & $6.5[3-165]$ \\
Re-operation & $10.5[4-133]$ \\
$30-$ day hospital readmission & 0 \\
$30-$ day mortality & 0 \\
\hline
\end{tabular}

*, data expressed as median and range.

Table 3 Pain score and analgesic requirement

\begin{tabular}{lc}
\hline Variables & $\mathrm{N}=8$ \\
\hline Pain score on discharge (at rest) & $0 / 10$ \\
Pain score on discharge (movement) & $\mathrm{O} / 10$ (range 0-2) \\
Analgesia on discharge & \\
None & 1 \\
Simple analgesia & 3 \\
Tramadol & 1 \\
Simple analgesia + tramadol & 2 \\
Simple analgesia + tramadol + gabapentin & 1 \\
\hline
\end{tabular}

*, data expressed as median and range.

were malignant infiltration of the chest wall in $50 \%$ of patients, 3 other subjects $(37.5 \%)$ had chest wall resections to achieve adequate surgical exposure and one patient had a right lung apical mycetoma with chest wall invasion (Table 2).

All patients underwent lung resections, right $(\mathrm{n}=6)$ and left $(\mathrm{n}=2)$, with a removal of 2 to 6 ribs (median 3$)$. Rib fixation was performed using the MatrixRib system, with a median of 2.5 ribs fixed (range, 2-4). A standard sized $10 \mathrm{~cm}$ by $15 \mathrm{~cm}$ Permacol patch was utilized and shaped to fit individually. One patient required the aid of a pedicaled muscle flap to fill up the thoracic cavity.

Superficial wound infection was observed in one patient and it resolved promptly with one week of oral antibiotics. There was no deep wound infection or seroma formation in this study. Amongst patients with malignancy who underwent chest wall reconstruction, one patient developed Enterobacter pneumonia which was successfully managed medically with a short course of intravenous piperacillin/ tazobactam. Upon discharge, the patient was prescribed with 3 weeks of oral ciprofloxacin and levofloxacin.

For the patients who underwent surgery with a preexisting lung infection, antibiotics were administered according to the recommendations of the infectious disease team. One subject was found to have non-tuberculous mycobacteria pulmonary infection (Mycobacterium kanasii). Incidentally, in the absence of deep seated wound infection, there was a screw dehiscence for that patient in the followup CT scan. This dehiscence did not appear to be related to the sepsis or lung infection. The risk factors of screw dehiscence in this patient include osteoporosis and surgical technique (multiple attempts of screw insertion into porous bone). This patient did not require implant removal.

On the day of discharge, the median pain score at rest and on movement was 0 out of 10 (Table 3). Analgesic requirements at discharge were minimal. $50 \%(n=4)$ required simple analgesia (paracetamol) or no analgesia. One patient was put on a low dose of tramadol as needed, due to paracetamol allergy. Another patient had a combination of simple oral analgesia with tramadol while another had simple analgesia, tramadol and gabapentin.

After discharge, the patients were reviewed in the outpatient clinic for at least 1 year. There was no patient mortality or hospital readmission for causes related to chest wall disease within 30 days. Representative postoperative CT and XR imaging at 3-6 months are shown in Figures 1-3. At 1 year, one patient with mesothelioma passed away from metastatic disease. The other patients were alive and asymptomatic for causes related to chest wall disease.

\section{Discussion}

The common indications for primary chest wall surgery include trauma, infection and malignancy (14), with the aim of preserving chest wall mechanics for physiological respiration, protecting vital structures and preventing organ 


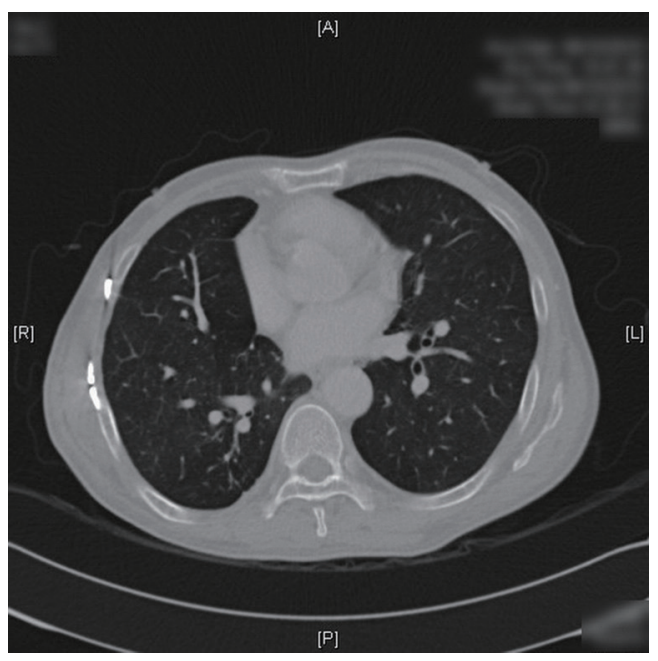

Figure 1 Postoperative CT thorax demonstrating preservation of right chest wall curvature after resection and fixation (axial view).

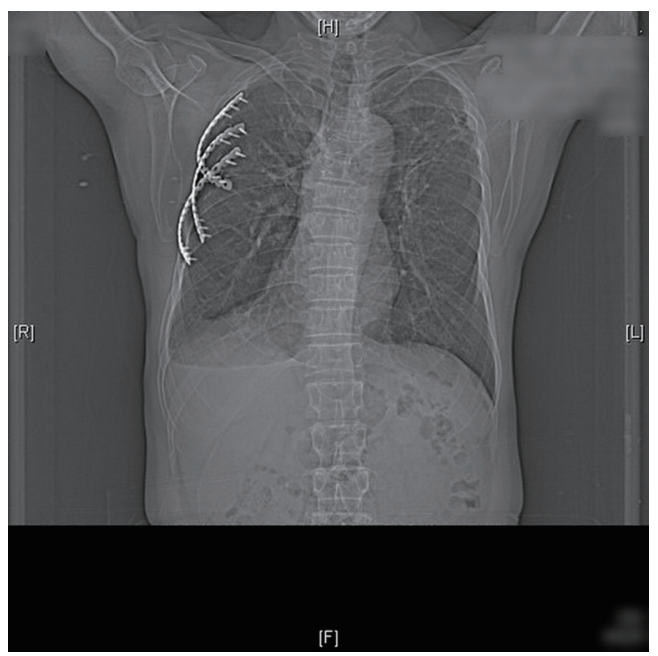

Figure 2 Postoperative CT thorax demonstrating preservation of right chest wall curvature after resection and fixation (coronal view).

herniation (4). The current consensus for reconstruction is when the defect is more than or equal to $5 \mathrm{~cm}$ or when the resection involves more than three ribs $(4,5,15)$. On the other hand, others advocate that almost all chest wall defects should be reconstructed, in light of the ease of repair and advances in chest wall repair techniques and materials (16). Some argue that it is unnecessary to repair defects beneath the scapula, in the apical-posterior aspect of the chest wall as there is adequate support from the shoulder and back

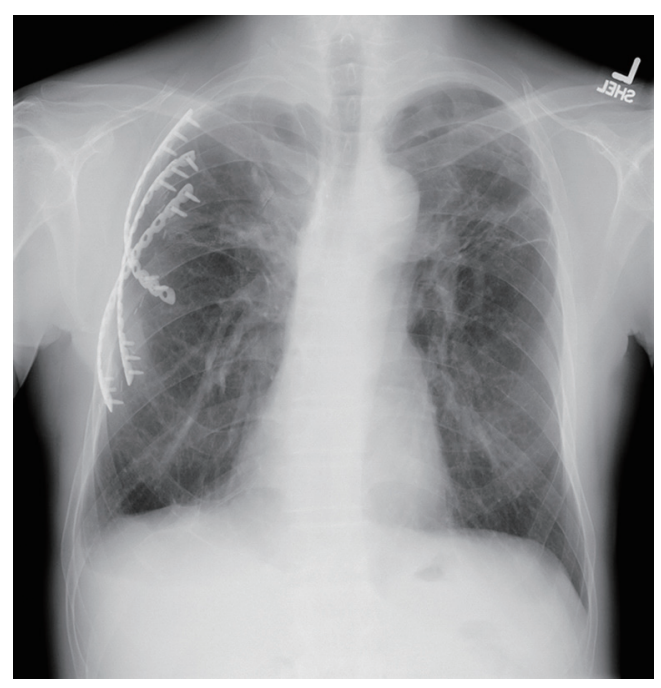

Figure 3 Postoperative chest X-ray showing well expanded right lung with preserved chest wall curvature and thoracic volume.

muscles (17). However, defects at the tips of the scapula should be addressed to prevent impingement of the internal structures of the thorax (18).

Historically, this type of surgery began as early as the 1940s, when Watson and James harvested biological fascia lata grafts for the repair of chest wall defects (19). Subsequently, different local and regional flaps (chest wall musculature and omentum) were utilized (4). However, this method inadvertently prolonged surgical time and resurfaced problems related to donor site morbidity and graft failure $(4,13)$. Moreover, there were issues with the conventional method of utilizing PTFE or polypropylene and methylmethacrylate. The use of methylmethacrylate was associated with fractures, chronic pain, rejection, infection and heat injury $(5,6)$. In addition, technical difficulties of handling and molding of the methacrylate often resulted in non-anatomical contours and reduction in intrathoracic volumes (5). There were also reports of seroma formation linked with the use of PTFE and polypropylene (6). Contaminated environments (infection or post radiotherapy necrosis) limited the application of synthetic materials as possible infection of the synthetic foreign material is a serious concern (19). When it occurred, these subjects ended up requiring prolonged intravenous antibiotics, removal of the infected synthetic material and multiple re-operations (19-21). Nevertheless, PTFE has been a useful synthetic material that is commonly applied in chest wall reconstruction $(6,7,21)$. 
It is water resistant, flexible and integrates with tissues. In combination with muscle flaps, the repair was only bidimensional with a loss in the curvature of the chest wall (6). This repair technique was optimized with the addition of titanium plates to maintain the shape of the chest wall $(5,6)$. Additionally, reports of chronic pain, adhesions, bleeding, fistula formation, lung injury and mesh shrinkage have been observed with the use of synthetic materials (1).

A host of new biomaterials (Permacol, porcine dermal collagen patch; Alloderm, acellular human dermal matrix; Veritas, bovine pericardial patches) and dedicated rib fixator implant systems (Synthes MatrixRib, STRATOS system, Biobridge) have been introduced into the field of chest wall reconstruction $(13,20)$. Miller and colleagues (20) demonstrated the safety and feasibility of the combination of Biobridge and Veritas for chest wall reconstruction in a contaminated field, where there was no removal of any materials from a contaminated site. During the followup review, paradoxical movement of the chest wall during respiration was absent in all subjects. Similarly, Barua and colleagues (13) showed satisfactory results with the Permacol and Veritas patches in contaminated sites (20).

Studies have demonstrated that titanium plates (Synthes MatrixRib, STRATOS system) were safe and effective for the bony reconstruction of chest wall defects $(5,6)$. They appeared to be rigid enough to prevent paradoxical movement of the chest wall and facilitate normal chest wall mechanics (22). Titanium is sufficiently inert to allow tissue integration, resistant to infection and is compatible with CT imaging with minimal interferences to permit visualization of the thorax post repair (22). Overall, the usage of these dedicated rib fixator systems appeared convenient and coupled with the distinct benefits discussed above, makes it a good option for the bony reconstruction of the chest wall $(3,23)$.

In our series, a total of 8 patients underwent chest wall reconstruction. With a median resection of 3 ribs, most subjects were left with a sizable chest wall defect. All defects were repaired as we believe that these patients would benefit in terms of chest wall mechanics, pain relief and protection of the visceral organs.

During the consideration of implanting foreign material into a clean-contaminated surgical field, Permacol was an attractive alternative as studies claimed that it was more resistant to infection because it promotes neovascularization, tissue regeneration and was observed to be absorbed fully at 1 year $(12,13)$. The current evidence is weak but logically it makes sense. Acting as a scaffold, the newly integrated tissue and vasculature will likely stand a higher chance against infection, as compared to their synthetic counterparts. The titanium based MatrixRib was utilized as it is relatively resistant to infection and does not affect CT imaging. This is an important feature especially for oncologic patients as they will almost definitely require repeat imaging post-surgery for surveillance. Likewise, we did not encounter any postoperative deep-seated infection or seroma formation apart from a superficial wound infection that was easily treated with a short course of oral antibiotics. This result is encouraging as implants were placed in clean-contaminated environments. The method which we employed to ensure anatomical restoration of the chest wall with MatrixRib appeared to work well as none of the patients had issues with the respiratory mechanics at the follow-up clinic. Post-operative CT scans also demonstrated anatomical restoration and conservation of the thoracic volumes in all subjects.

In addition, we believe that the near-zero pain score and minimal oral analgesic requirements in our cohort of patients can be attributed to anatomical restoration of the chest wall defect achieved via specific surgical techniques as discussed in the Methods section. The thoracotomy approach was employed for all subjects to obtain adequate exposure for extensive resection of the chest wall and intra-thoracic structures. Minimally invasive techniques were unsuitable for this cohorts of patients. The number of titanium bars utilized was equal to the number of ribs reconstructed. Studies have shown that ribs 4 to 10 are the most mobile and commonly repaired (24). We believe ribs 5 to 8 were most important in respiratory mechanics of the chest wall, hence, not all costal segments were reconstructed. The titanium bars were also used as a prosthetic bridge to provide stability for the thoracotomy incision. This method preserved the rigidity and functionality of the chest wall. There were no implant failures or fractures in our study except for a screw dehiscence in one patient. As the chest wall was stable and the patient remained asymptomatic, the screw dehiscence was managed conservatively. In addition, operative removal entailed a higher surgical risk than the benefit provided by removal of the affected titanium bar or screw. None of the subjects required removal of the implants. MatrixRib appeared to be a reliable system in the repair of the rigid component of the chest wall.

Our study has a number of limitations. It is a retrospective analysis of a single institutional practice with no control group. The sample size of this feasibility study is small with a heterogenous group of subjects. The follow-up duration of 1 
year is insufficient to evaluate the long-term outcomes of this technique. However, there was no selection bias as these were consecutive repairs of chest wall defects at our institution. In addition, post-operative lung function tests were not performed as our study population represents a heterogenous group of patients with parenchymal lung diseases (malignancy and infection), all of whom underwent varying degrees of lung resection. Thus, post-operative lung function test would not be reflective of the chest wall function alone.

In conclusion, this study provides confidence in the safety and feasibility of coupling the MatrixRib system and Permacol patch for chest wall reconstruction. Our initial experience with this versatile combination is promising in terms of resistance to infection in clean-contaminated environments. More uniquely, this novel combination seemed to facilitate a pain-free experience for the patients. Future studies are needed to investigate the long-term safety and efficacy of this repair technique.

\section{Acknowledgements}

None.

\section{Footnote}

Conflicts of Interest: The authors have no conflicts of interest to declare.

Ethical Statement: This study has been approved by the Domain Specific Review Board National Health Care Group (Singapore) (No. 2017/00607), with waiver of patient consent.

\section{References}

1. Wiegmann B, Zardo P, Dickgreber N, et al. Biological materials in chest wall reconstruction: initial experience with the Peri-Guard Repair Patch ${ }^{\circledR}$. Eur J Cardiothorac Surg 2010;37:602-5.

2. Iarussi T, Pardolesi A, Camplese P, et al. Composite chest wall reconstruction using titanium plates and mesh preserves chest wall function. J Thorac Cardiovasc Surg 2010;140:476-7.

3. Ng CS. Recent and Future Developments in Chest Wall Reconstruction. Semin Thorac Cardiovasc Surg 2015;27:234-9.

4. Momeni A, Kovach SJ. Important considerations in chest wall reconstruction. J Surg Oncol 2016;113:913-22.
5. Billè A, Okiror L, Karenovics W, et al. Experience with titanium devices for rib fixation and coverage of chest wall defects. Interact Cardiovasc Thorac Surg 2012;15:588-95.

6. Berthet JP, Canaud L, D'Annoville T, et al. Titanium plates and Dualmesh: a modern combination for reconstructing very large chest wall defects. Ann Thorac Surg 2011;91:1709-16.

7. Berthet JP, Wihlm JM, Canaud L, et al. The combination of polytetrafluoroethylene mesh and titanium rib implants: an innovative process for reconstructing large full thickness chest wall defects. Eur J Cardiothorac Surg 2012;42:444-53 .

8. Bachman S, Ramshaw B. Prosthetic material in ventral hernia repair: how do I choose? Surg Clin North Am 2008;88:101-12, ix.

9. Hiles M, Record Ritchie RD, Altizer AM. Are biologic grafts effective for hernia repair? A systematic review of the literature. Surg Innov 2009;16:26-37.

10. Shankaran V, Weber DJ, Reed RL 2nd, et al. A review of available prosthetics for ventral hernia repair. Ann Surg 2011;253:16-26.

11. Alaedeen DI, Lipman J, Medalie D, et al. The single stage approach to the surgical management of abdominal wall hernias in contaminated fields. Hernia 2007;11:41-5.

12. Peacock O, Pandya H, Sharp T, et al. Biological mesh reconstruction of perineal wounds following enhanced abdominoperineal excision of rectum (APER). Int J Colorectal Dis 2012;27:475-82 .

13. Barua A, Catton JA, Socci L, et al. Initial experience with the use of biological implants for soft tissue and chest wall reconstruction in thoracic surgery. Ann Thorac Surg 2012;94:1701-5.

14. Clemens MW, Evans KK, Mardini S, et al. Introduction to Chest Wall Reconstruction: Anatomy and Physiology of the Chest and Indications for Chest Wall Reconstruction. Semin Plast Surg 2011;25:5-15.

15. Gonfiotti A, Santini PF, Campanacci D, et al. Malignant primary chest-wall tumours: techniques of reconstruction and survival. Eur J Cardiothorac Surg 2010;38:39-45.

16. Rocco G: Chest wall resection and reconstruction according to the principles of biomimesis. Semin Thorac Cardiovasc Surg 2011;23:307-13.

17. Seder CW, Rocco G. Chest wall reconstruction after extended resection. J Thorac Dis 2016;8:S863-S871.

18. Watson WL, James AG. Fascia lata grafts for chest wall defects. J Thorac Surg 1947;16:399-406.

19. Deschamps C, Tirnaksiz BM, Darbandi R, et al. Early and long-term results of prosthetic chest wall 
reconstruction. J Thorac Cardiovasc Surg 1999;117:58891; discussion 591-2.

20. Miller DL, Force SD, Pickens A, et al. Chest wall reconstruction using biomaterials. Ann Thorac Surg 2013;95:1050-6.

21. Weyant MJ, Bains MS, Venkatraman E, et al. Results of chest wall resection and reconstruction with and without rigid prosthesis. Ann Thorac Surg 2006;81:279-85.

22. Coonar AS, Wihlm JM, Wells FC, et al. Intermediate outcome and dynamic computerised tomography after

Cite this article as: Ong K, Ong CS, Chua YC, Fazuludeen AA, Ahmed AD. The painless combination of anatomically contoured titanium plates and porcine dermal collagen patch for chest wall reconstruction. J Thorac Dis 2018;10(5):28902897. doi: $10.21037 /$ jtd.2018.04.116 chest wall reconstruction with the STRATOS titanium rib bridge system: video demonstration of preserved buckethandle rib motion. Interact Cardiovasc Thorac Surg 2011;12:80-1.

23. Bottlang M, Long WB, Phelan D, et al. Surgical stabilization of flail chest injuries with MatrixRIB implants: a prospective observational study. Injury. 2013;44:232-8.

24. de Moya M, Nirula R, Biffl W. Rib fixation: Who, What, When? Trauma Surgery \& Acute Care Open 2017;2:e00059. 